\title{
Experimental and Analytical Study of Free-Fall Drop Impact Testing of Portable Products
}

\author{
E. Tempelman • M.M.S. Dwaikat • C. Spitás
}

Received: 10 August 2011 / Accepted: 16 December 2011 / Published online: 26 January 2012

(C) The Author(s) 2012. This article is published with open access at Springerlink.com

\begin{abstract}
Portable products can suffer critical damage due to drop impact and thus, such load cases must be taken into account in the conceptual and detailed design phases of such products. One method explored in the current study for alleviating the peak accelerations resulting from impact is to isolate fragile components from the product housing using internal shock mounts. There is a lack of studies that address this method in a systematic and reliable manner. This paper presents an experimental and analytical study on the dynamic behaviour during impact of portable products with internal shock mounting. Using a special drop tower with guiding frame for controlled-angle free-fall drop impact, representative products are dropped at different angles and the acceleration is recorded both on the outer case and on an internally-mounted plate. A simplified analytical procedure, suitable for conceptual design purposes, is proposed for predicting the resulting dynamic response. The work affirms the suitability of guide-and-release facilities in impact experiments; additionally, it may facilitate the conceptual design for impact resistance of portable products.
\end{abstract}

Keywords Drop testing · Portable products · Dynamic analysis $\cdot$ Natural frequency $\cdot$ Impact loading

\section{Introduction}

So far, the behaviour of consumer electronics under sudden shocks or impacts has eluded full understanding, despite the

E. Tempelman $(\varangle) \cdot$ M.M.S. Dwaikat $\cdot$ C. Spitás

Faculty of Industrial Design Engineering,

Delft University of Technology,

Landbergstraat 15 ,

2628 CE, Delft, the Netherlands

e-mail: e.tempelman@tudelft.nl fact that impact is a major cause of premature failure for such products - especially for handheld electronics. As a result, manufacturers of cell phones, shavers etc. still have to resort to time-consuming design-failure-redesign cycles $[1,2]$.

Much of current research on impact concerns product components, such as soldered joints (see e.g. [3, 4]), LCD's (e.g. [5]) or PCB's (notably [6]). Publications involving full products are more scarce: e.g. Lim et al. [7] performed drop impact tests on cell phones and PDA's, but without analysis; Singh et al. [8] combined analysis with simulations, but performed no experimental validation; Zhou et al. [6] did both. Such studies are useful for examining the effects of small, 'steel safe' changes late in the product development process, but they are not directly suitable for supporting choices at earlier stages (e.g. shape, basic lay-out, size). Apart from that, the complexities of real product geometries obscure the analytical view that is eventually indispensable to understanding.

The authors therefore commenced studying product impact in a systematic manner, designing simplified products that lend themselves equally well to analysis as to experiment. This research focuses on the role of internal shock mounts used to isolate fragile components from the outer product case, thus reducing the peak accelerations these components experience due to impact. Such base-isolation is investigated here by mounting fragile components on rubber cubes. The analysis was carried out using elastic impact theory, rigid body kinematics, and dynamics equations. Experiments were carried out using a dedicated drop test facility comparable to the one put forward by Goyal and Buratynski [9]. Our test facility employs a guiding frame to allow free-fall drop testing of products with accurate control over the contact angle (i.e. the angle between the ground and a suitable product reference line), even with built-in wire-based sensors. 


\section{Theory}

Impact Speed, Contact Time, Peak Deceleration

Since this study aims to generate knowledge that is applicable during the conceptual design phase of product development (apart from the experimental work itself), a simplified analysis procedure will be followed. For products in free-fall, the initial impact velocity $v_{i}$ follows from the basic laws of conservation of energy, assuming that air resistance is negligible, which is valid for speeds below $10 \mathrm{~m} / \mathrm{s}$ and relatively compact products. Given a certain rebound height $Y_{r}$ we obtain the total velocity change experienced by the product as follows: $\Delta v=$ $\left(1+e_{c}\right) \sqrt{2 g Y}$ with $Y$ being the drop height, $g$ the standard gravity of $9.81 \mathrm{~m} / \mathrm{s}^{2}$ and $e_{c}$ is the rebound ratio $\sqrt{Y_{r} / Y}$. Typically, for low-speed impact, peak decelerations are in the range of hundreds of $g$ 's, and contact times in the range of milliseconds.

What happens during the contact time and immediately afterwards can be analysed in the light of rigid body dynamics, Hertzian contact theory and the impulse dynamics of the system. Specifically, the main test geometry, consisting of a box case with an internal plate mounted on rubber cubes (further detailed under Experimental Procedure as 'geometry-2'), will be considered as one rigid body box system from the initiation of impact (at $t=0$ ) onwards. Rigid body dynamics will be utilized to compute the total vertical acceleration of the box contact point as a function of the impact force. Next, Hertzian non-adhesive elastic contact theory will be employed to compute the impact force, contact time, and compressed depth of contact. Finally, impulse dynamics will be used to compute the response of the box system and the internally-mounted plate.

\section{System Kinetics}

Let us consider the dropped box system as one rigid body system landing on its corner as shown in Fig. 1. This system

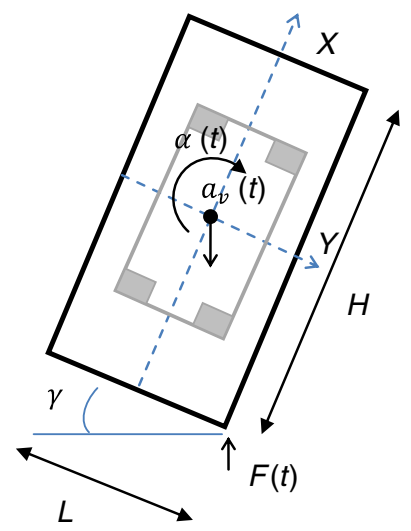

Fig. 1 Kinetics of the box system under drop impact can be idealized as a rigid body with vertical $a_{v}(t)$ and angular $\alpha(t)$ accelerations at its center of mass. The angular acceleration results from the impact force $F$ at the point of contact. For the given product, the centre of stiffness coincides with the centre of mass and rotational acceleration is taken to be around it. Applying the Euler-Newton law of angular equilibrium at the centre of mass we obtain:

$\sum r \times F=I_{p} \alpha(t)$

Here, $I_{p}=m\left(H^{2}+L^{2}\right) / 12$ is the angular mass moment of inertia for a rectangular plate with mass $m$ and dimensions $L \times H$. Applying equation (1) at the centre gives the following relation for the angular acceleration:

$\alpha(t)=\frac{F(t)}{I_{p}}\left(\frac{L}{2} \cos (\gamma)-\frac{H}{2} \sin (\gamma)\right)$

Using equation (2) with the vertical translational acceleration $a_{v}(t)=F(t) / m$, the total vertical acceleration at the point of contact can then obtained as:

$\alpha_{T}=\frac{d^{2} \delta}{d t^{2}}=\alpha_{v}(t)+\alpha(t)\left(\frac{L}{2} \cos (\gamma)-\frac{H}{2} \sin (\gamma)\right)$

$\frac{d^{2} \delta}{d t^{2}}=\frac{F(t)}{m /\left\{1+\left(\frac{3}{2}\right) \frac{\cos ^{2}(\gamma)[1-(H / L) \tan (\gamma)]^{2}}{1+(L / H)^{2}}\right\}}$

Here, $\delta$ is the compressed depth of contact, while the denominator of equation ( $3 \mathrm{~b}$ ) will henceforth be called the 'equivalent mass' $m_{e}$ :

$m_{e}=\frac{m}{1+\left(\frac{3}{2}\right) \frac{\cos ^{2}(\gamma)[1-(H / L) \tan (\gamma)]^{2}}{1+(L / H)^{2}}}$

Impact Force and Impulse

Since the contact area is very small compared to the box system and the impact speed is low $(<10 \mathrm{~m} / \mathrm{s})$, Hertzian impact theory can be used to approximate the resulting impact force and contact time [6, 10]. Accordingly, the impact force $F(t)$ is a highly nonlinear function of $\delta$ :

$F(t)=m_{e} \frac{d^{2} \delta}{d t^{2}}=\delta K \sqrt{\delta R}$

Here, $R$ is the radius of the impacting region, $m_{e}$ is the equivalent mass of the system and $K$ is indicative of the 'contact stiffness' given as:

$K=\frac{4 / 3}{\frac{1-\mu_{i}^{2}}{E_{i}}+\frac{1-\mu_{s}^{2}}{E_{s}}}$ 
In this equation, $\mu_{i, s}$ and $E_{i, s}$ are the Poisson's ratio and Young's modulus of the impactor and the impacted surface, respectively. Analytical solution for equation (5) is impossible due to the high nonlinearity, but Hunter [11] provides an approximation for $\delta$ and $F$ as a semi-sinusoidal progression over the contact time $T_{i}$ :

$\delta(t)=\delta_{\max } \sin \left(\pi \frac{t}{T_{i}}\right)=\left(\frac{5 m_{e} v_{i}^{2}}{4 K \sqrt{R}}\right)^{\frac{2}{5}} \sin \left(\pi \frac{t}{T_{i}}\right)$

and

$F(t)=m_{e}\left(\frac{\pi}{T_{i}}\right)^{2} \delta(t), t \leq T_{i}$

Here, $v_{i}$ is the initial impact velocity and $T_{i}$ is the contact time computed as:

$T_{i}=2.943 \frac{\delta_{\max }}{v_{i}}$

Once the impact force is known, the impulse due to impact can then be computed as the integral of the impact force over the contact time. By decomposing the impact force $F(t)$ into two components along the $\mathrm{X}$ - and $\mathrm{Y}$-axes of the box system, two equations can be written for the impulse:

$\mathbb{\square}_{X}=\cos (\gamma) \int_{0}^{T_{i}} F(t) d t=m_{B}\left\{\dot{w}_{X}\left(t=T_{i}\right)-v_{i} \cos (\gamma)\right\}$

$\rrbracket_{Y}=\sin (\gamma) \int_{0}^{T_{i}} F(t) d t=m_{B}\left\{\dot{w}_{Y}\left(t=T_{i}\right)-v_{i} \sin (\gamma)\right\}$

Here, $\square_{X}$ and $\rrbracket_{Y}$ represent the $\mathrm{X}$ and $\mathrm{Y}$ components of the total impulse. $m_{B}$ and $w_{X Y}$ are the mass and the displacements of the box system, respectively. The decomposition of the impulse into $\mathrm{X}$ and $\mathrm{Y}$ components is due to the fact that the box landed vertically (zero inclination in the $\mathrm{z}$-direction) at point of contact If the box XY plane is inclined towards the $\mathrm{z}$-direction, then a third component of impulse $\left(\square_{Z}\right)$ needs to be computed in the same fashion as in equations $(10 \mathrm{a}, 10 \mathrm{~b})$. Care should be taken for the computation of natural frequency $\omega_{p}$ of the plate in the out-of-plane direction.

\section{System Dynamics: Box Case}

The box system, that was considered above as a rigid body during impact to obtain impact force and duration, will be assumed to be a single mass that is vibrating with a frequency similar to that of the impacting force (i.e., $\omega_{B}=\pi / T_{i}$ ). Based on this, two equations of motion (in X- and Y-directions) for the box subjected to impulses given in equations $(10 \mathrm{a}, 10 \mathrm{~b})$ can be written [12]:

$w_{X}+2 \omega_{B} \xi_{B} \dot{w}_{X}+\omega_{B}^{2} w_{X}=\frac{\rrbracket_{X}}{m_{B}} \cdot \delta^{D}\left(t-T_{i}\right)$

$w_{Y}+2 \omega_{B} \xi_{B} \dot{w}_{Y}+\omega_{B}^{2} w_{Y}=\frac{\rrbracket_{Y}}{m_{B}} \cdot \delta^{D}\left(t-T_{i}\right)$

Here, $w_{X, Y}$ are the displacements of the box in the X-and Y-directions, $\omega_{B}$ and $\xi_{B}$ are the natural frequency and the damping ratio of the box $\left(\xi_{B}=\frac{c_{B}}{2 \omega_{B} m_{B}}\right)$ and $\delta^{D}\left(t-T_{i}\right)$ is the Dirac Delta-Function defined as follows:

$\delta^{D}\left(t-T_{i}\right)=\left\{\begin{array}{cr}0, & t<T_{i} \text { and } t>T_{i} \\ \int_{-\infty}^{\infty} \delta^{D}\left(t-T_{i}\right) d t=1, t=T_{i}\end{array}\right.$

For solving equations $(11 \mathrm{a}, 11 \mathrm{~b})$ the following boundary conditions apply:

For Eq. $11 a: w_{X}\left(t=T_{i}\right)=0$ and $\dot{w}_{X}\left(t=T_{i}\right)=\frac{\mathbb{Q}_{X}}{m_{B}}+v_{i} \cos (\gamma)$

For Eq. $11 b: w_{Y}\left(t=T_{i}\right)=0$ and $\dot{w}_{Y}\left(t=T_{i}\right)=\frac{\square_{Y}}{m_{B}}+v_{i} \sin (\gamma)$

Next, the damped transient solutions of equations (11a, 11b) can be obtained as:

$\left[\begin{array}{c}w_{X} \\ w_{Y}\end{array}\right]=\left[\begin{array}{c}\frac{\square_{X}}{m_{B}}+v_{i} \cos (\gamma) \\ \frac{D_{Y}}{m_{B}}+v_{i} \sin (\gamma)\end{array}\right] \cdot \frac{e^{-\xi_{B} \omega_{B}\left(t-T_{i}\right)}}{\omega_{B, D}} \cdot \sin \left(\omega_{B, D}\left(t-T_{i}\right)\right)$

Here, $\omega_{B, D}=\omega_{B} \sqrt{1-\xi_{B}^{2}}$ is the damped natural frequency of the box system. Differentiating these solutions will result in its accelerations in the two directions.

\section{System Dynamics: Internally Mounted Plate}

The mounted plate will be considered as a SDOF that is subjected to sinusoidal ground motion $w_{X, Y}=$ $A_{X, Y} \sin \left(\omega_{B, D}\left(t-T_{i}\right)\right)$ during impact, where $A_{X, Y}$ are the maximum displacements (in $\mathrm{X}$ - and $\mathrm{Y}$-directions) of the box system and can be found in equation (14). This idealization is shown in Fig. 2, and it is valid as long as $t<T_{i}$. However, after impact $(t>T i)$ the mounted plate will oscillate under free damped vibration, since the box case acceleration $\ddot{w}_{X, Y}$ is 


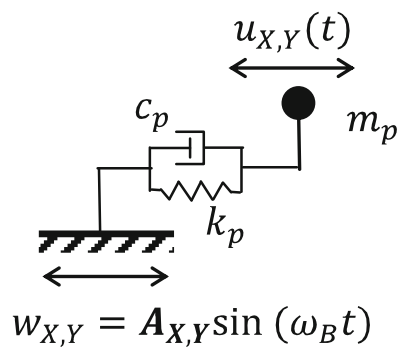

Fig. 2 Idealization of the rubber-cube mounted plate inside the stiff box case

assumed to have vanished for $t>T_{i}$. (As will be shown, this assumption is consistent with the observed results for the box system.) The equation of motion of the mounted plate becomes:

$m_{p} \ddot{u}_{X, Y}=c_{p}\left(\dot{w}_{X, Y}-\dot{u}_{X, Y}\right)+k_{p}\left(w_{X, Y}-u_{X, Y}\right)$ For $t<T_{i}$

This can be rewritten into:

$\ddot{u}_{X, Y}+2 \omega_{p} \xi_{p} \dot{u}_{X, Y}+\omega_{p}^{2} u_{X, Y}=2 \omega_{p} \xi_{p}\left(\dot{w}_{X, Y}-\dot{u}_{X, Y}\right)+\omega_{p}^{2} w_{X, Y}$

$\ddot{u}_{X, Y}+2 \omega_{p} \xi_{p} \dot{u}_{X, Y}+\omega_{p}^{2} u_{X, Y}=\omega_{p}^{2} A \sqrt{1+\left(2 \xi_{p} \omega_{B} / \omega_{p}\right)^{2}} \sin \left(\omega_{B} t+\alpha\right)$

Here, $\alpha=\tan ^{-1}\left(2 \xi_{p} \omega_{B} / \omega_{p}\right)$, while $u_{X, Y}, \omega_{p}$ and $\xi_{p}$ are $\mathrm{X}$ and $\mathrm{Y}$ displacements, the natural frequency and the damping ratio of the mounted plate respectively. Assuming the mounted plate was initially at rest with respect to the box system, the solution to the above differential equations can be obtained as [12]:

$$
u_{X, Y}(t)=\frac{A_{X, Y} \sin \left(\omega_{B, D} t+\alpha-\psi\right)}{\sqrt{1-\frac{2-\left(\omega_{B, D} / \omega_{p, D}\right)^{2}}{4 \xi_{p}^{2}+\left(\omega_{p, D} / \omega_{B, D}\right)^{2}}}} \text { For } t<T_{i}
$$
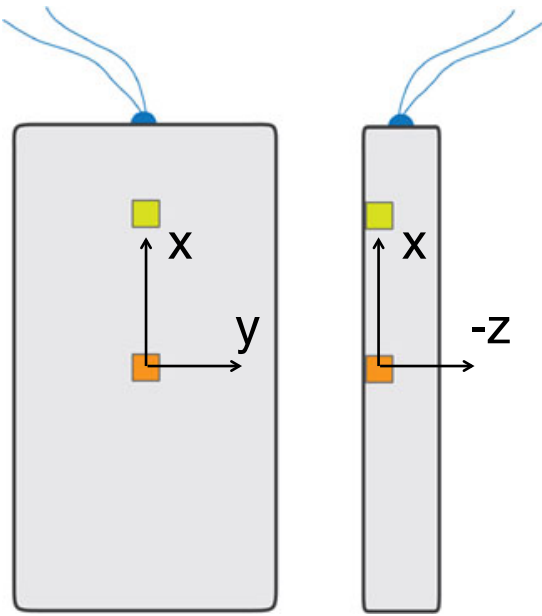

Figs. 3 Test geometry 1
Here, $\quad \psi=\tan ^{-1}\left(2 \xi_{p} \frac{\omega_{B, D} / \omega_{p, D}}{1-\left(\omega_{B, D} / \omega_{p, D}\right)^{2}}\right)$ and $\omega_{p, D}=$ $\omega_{p} \sqrt{1-\xi_{p}^{2}}$. Immediately after the contact time $\left(t>T_{i}\right)$, the mounted plate will undergo a free damped vibration with an eigen frequency $\omega_{p, D}$.

$u_{X, Y}^{\text {free }}(t)=e^{-\xi_{p} \omega_{p} t} u_{X, Y}(t) \quad$ For $t>T_{i}$

\section{Experimental Procedure}

\section{Design-Of-Experiment: Test Geometries}

As the basis for a representative product, a standard commercially-available flat ABS injection moulded box was selected, measuring $289 \times 101 \times 21 \mathrm{~mm}$, with a $2.0 \mathrm{~mm}$ wall thickness and rounded corners (Fig. 3). It consists of two halves with small screw bosses in each corner, held together with four M2.5 screws. The edges have a tongueand-groove joint over the thickness, but otherwise the box has no internal ribs or other irregularities. Its empty weight is 165 gr.

Using such a box, test geometry-1 was designed, having two accelerometers placed inside: S1 at the centre of gravity (COG) and S2 placed $65 \mathrm{~mm}$ higher (Fig. 3). Both sensors are tri-axial, positioned such that their $\mathrm{X}$-axes coincided with the length, Y-axes with the width and Z-axes with the thickness of the box. Geometry-1 was the reference point for the research. Test geometries-2A and $-2 \mathrm{~B}$ were designed using two additional ABS boxes. In both, $\mathrm{S} 1$ was mounted onto a $2.0 \mathrm{~mm}$ thick ABS plate measuring 100 x $50 \mathrm{~mm}$ centred inside the box (Fig. 4). This 10-gr plate was mounted on 3-mm (geometry-2A) or 5-mm (geometry-2B) solid rubber cubes, acting as internal shock mounts. Both times, S2 was placed in the same location as in geometry-1,

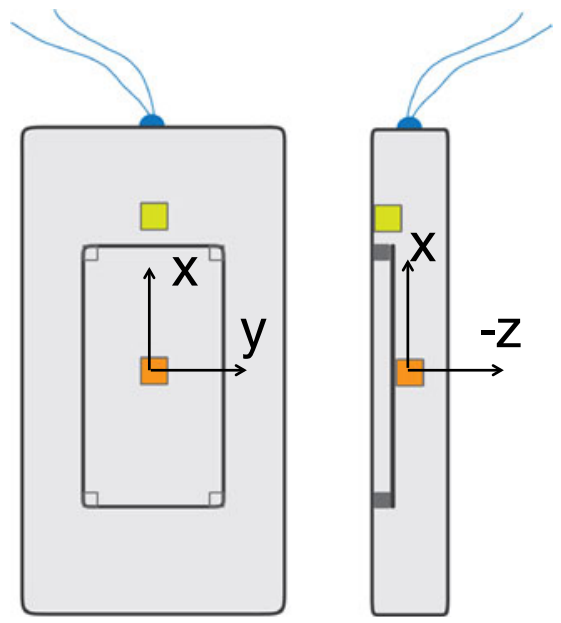

Fig. 4 Test geometries 2A and 2B 
Fig. 5 Lay-out of drop test facility used in this research

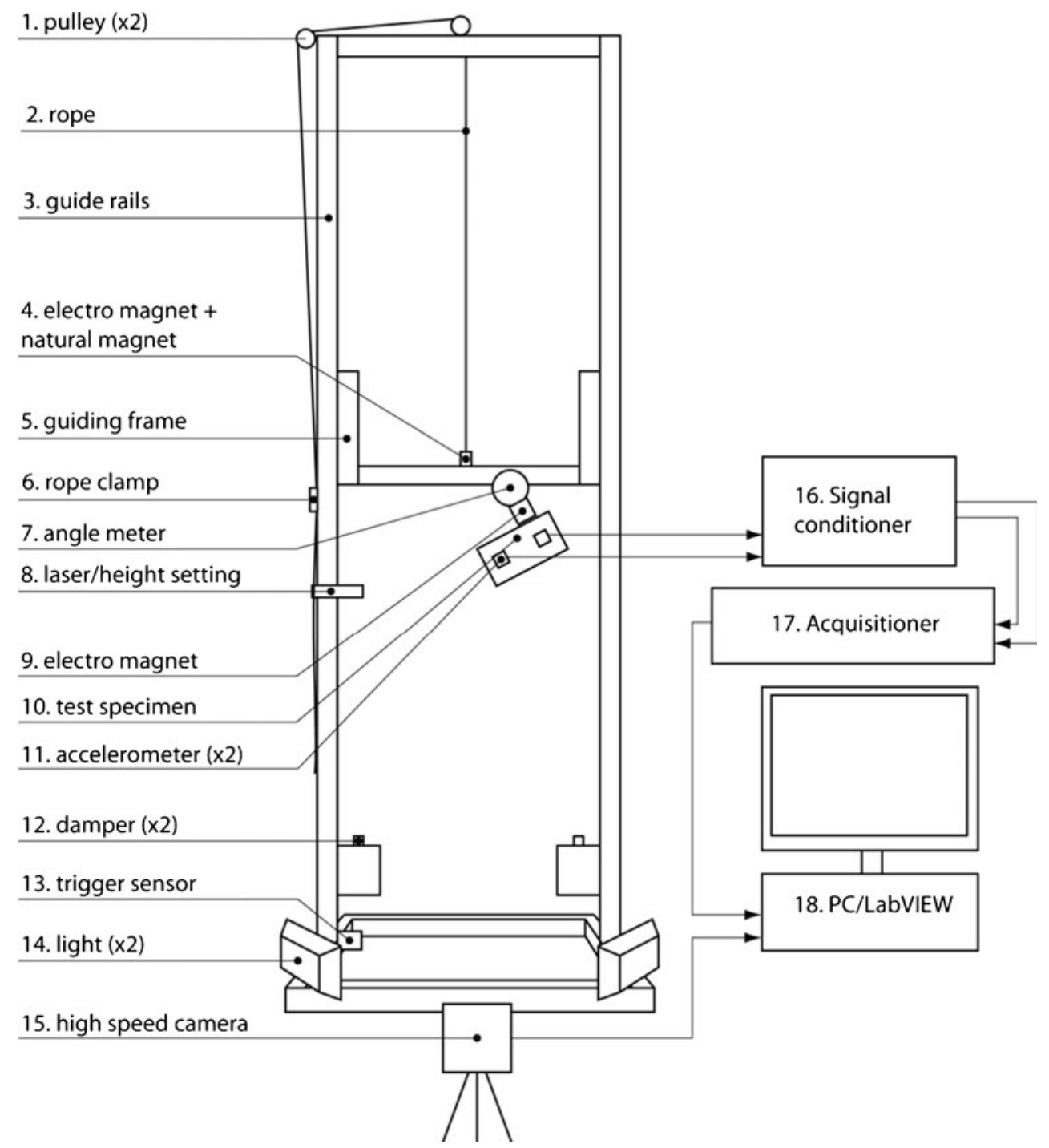

allowing comparison of results. Geometries- 2 served to determine the efficiency of the chosen types of internal shock mounting. They should be considered as simplified products with sufficient complexity to merit attention (of course, in actual products, more sophisticated shock mount shapes would be chosen, but cubes are both easy to model and feasible to accurately prototype on the basis of 3- and 5-mm thick sheets).

\section{Material Characterization}

If we assume the mounted plate to behave as infinitely rigid, then the lateral stiffness of the mounts $k_{p}$ (in equation (15)) plays a key role in reducing peak accelerations on the plate in geometries-2. Therefore, it was decided to measure the force-displacement curves in the XY plane separately, using a test bench to fix the (opened) box and pulling on the S1 sensor mount with a force gauge at different orientations in the plane, varying $\gamma$ between $0-90^{\circ}$. Also, the ABS from the boxes was characterized by taking out two standard ISO 527 type-1 test bars from a separate box and performing tensile testing.

Drop Test Facility and Set-up

The drop tests described here were done using a dedicated 'drop tower' shown in Fig. 5. It enables free-fall drop testing of small- to medium-sized products from heights of 0.60 to $2.40 \mathrm{~m}$ onto any surface as desired. Impact is recorded using a high speed video camera; furthermore, one or more sensors can be placed in- or on the product, as described earlier. The tower has two vertical guiderails along which a support frame glides on low-friction bearings. This frame serves as an attachment point for the sensor wires emanating from the product so that product and wires are brought to the same speed during the drop (Fig. 6).

Just before impact, the frame is brought to a stop using two dampers, with the slack in the wires allowing the 


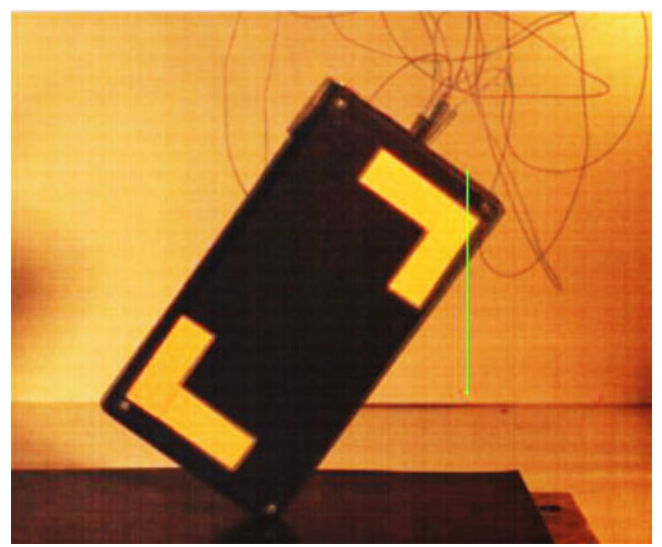

Fig. 6 Five overlapping images for geometry- 1 at $35^{\circ}$, taken midway during the contact time (except for the sensor wires, the box was in exactly the same orientation each time)

product to continue independently of the frame. The product itself is attached to the frame via an electromagnet (to this end, a tiny steel strip is glued to the product) and is released just before the frame is stopped. This arrangement keeps the sensor wires from interfering with the drop itself and enables accurate control over the contact angle. Computer linkups and customized software allow easy synchronisation of all data streams and logging of sensor data (i.e. acceleration histories and peak values) as well as drop tower operation in general. In particular, the contact angle can be checked visually.

For all tests, two tri-axial ceramic shear ICP ${ }^{\circledR}$ accelerometers, procured from PCB Piezotronics were used. These sensors can accurately measure accelerations up to $4905 \mathrm{~m} / \mathrm{s}^{2}$ $(500 \mathrm{~g})$ with sampling frequencies of $10 \mathrm{kHz}(\mathrm{Y} / \mathrm{Z}$ axes) and $7 \mathrm{kHz}$ ( $\mathrm{X}$ axis). Their size is one cubic centimetre; weight (excluding wire) is $4 \mathrm{gr}$. High speed videos were made using a Minivis Speedcam procured form Weinberger, at 1,000 fps (640 by 512 pixel image).
Test Procedure

All geometries were dropped onto a $10 \mathrm{~mm}$ thick shore 40 rubber mat, with a drop height $Y$ of $0.894 \mathrm{~m}$. The angle between the vertical and the $\mathrm{X}$-axis of the product (i.e. the contact angle) was set at $15^{\circ}, 20^{\circ}, 25^{\circ}, 30^{\circ}$ and $35^{\circ}$; note that at $25^{\circ}$, the COG lies exactly above the contact point. Each test was executed five times. Each time, the HS video footage was observed and any suspect measurements (e.g. with unrealistic $Z$-accelerations, visibly tangling wires) were redone. Apart from $\mathrm{X}$ - and $\mathrm{Y}$-accelerations, the combined vector was determined also, checking the acceleration histories to ensure that the peak values in both directions were reached at the same instant. Test reproducibility i.e. angle control was also checked separately by overlapping the HS images captured at the moment of impact from the five tests in each series. Furthermore, in a series of additional tests with the guidance frame fixed in the uppermost position, the rebound height $Y_{r}$ was determined through observation by dropping an empty ABS box.

\section{Experimental Results}

For geometry-1, the sensor data are given in Table 1, with typical acceleration histories for S1 (centred inside case, at $\mathrm{COG}$ ) and S2 (near top inside case, $65 \mathrm{~mm}$ up from $\mathrm{COG}$ ) given in Figs. 7, 8 and 9. From observation of recorded HS video, contact time was found to be $3 \pm 0.2 \mathrm{~ms}$. Furthermore, the histories show that build-up of force over the contact time on the box case is roughly sinusoidal (Fig. 7 right side: white (X-axis) and red (Y-axis) graphs). The time interval between the two sensor peaks was found to be very short and could not be reliably measured. (This observation encourages us to assume rigid-body behaviour for the case.)

Table 1 Sensor data, geometry-1

\begin{tabular}{|c|c|c|c|c|c|c|c|c|c|c|}
\hline & & \multicolumn{4}{|c|}{ Sensor-1 (centred inside case) } & \multicolumn{4}{|c|}{ Sensor-2 (near top inside case) } & \multirow[t]{2}{*}{ unit } \\
\hline & & $\mathrm{X}$ & $\mathrm{Y}$ & $\sqrt{ }\left(X^{2}+Y^{2}\right)$ & Z & $\mathrm{X}$ & $\mathrm{Y}$ & $\sqrt{ }\left(X^{2}+Y^{2}\right)$ & $\mathrm{Z}$ & \\
\hline \multirow[t]{2}{*}{ angle 15} & mean & $-578,7$ & $-191,1$ & 609,4 & $-13,5$ & $-590,6$ & $-271,6$ & 650,1 & 27,6 & g \\
\hline & SD & 25,9 & 22,9 & & 12,3 & 34,6 & 28,9 & & 11,9 & $\mathrm{~g}$ \\
\hline \multirow[t]{2}{*}{ angle 20} & mean & $-500,4$ & $-213,2$ & 543,9 & $-13,3$ & -509 & $-243,3$ & 564,2 & 21,5 & $\mathrm{~g}$ \\
\hline & SD & 13,6 & 15,8 & & 2,4 & 8,3 & 5,4 & & 2,0 & $\mathrm{~g}$ \\
\hline \multirow[t]{2}{*}{ angle 25} & mean & $-465,1$ & $-188,2$ & 501,7 & $-19,8$ & $-453,4$ & $-206,2$ & 498,1 & 21,2 & $\mathrm{~g}$ \\
\hline & SD & 6,7 & 6,4 & & 2,5 & 5,1 & 5 & & 1,9 & $\mathrm{~g}$ \\
\hline \multirow[t]{2}{*}{ angle 30} & mean & -445 & $-234,6$ & 503,1 & $-9,0$ & $-440,6$ & $-202,1$ & 484,7 & 21,0 & $\mathrm{~g}$ \\
\hline & SD & 6,3 & 3,2 & & 4,7 & 3,8 & 1,9 & & 0,3 & $\mathrm{~g}$ \\
\hline \multirow[t]{2}{*}{ angle 35} & mean & $-424,3$ & $-254,4$ & 494,7 & $-7,0$ & $-414,1$ & $-198,1$ & 459,0 & 19,2 & $\mathrm{~g}$ \\
\hline & SD & 6,4 & 2,1 & & 2,9 & 7,2 & 1,8 & & 1,7 & g \\
\hline
\end{tabular}




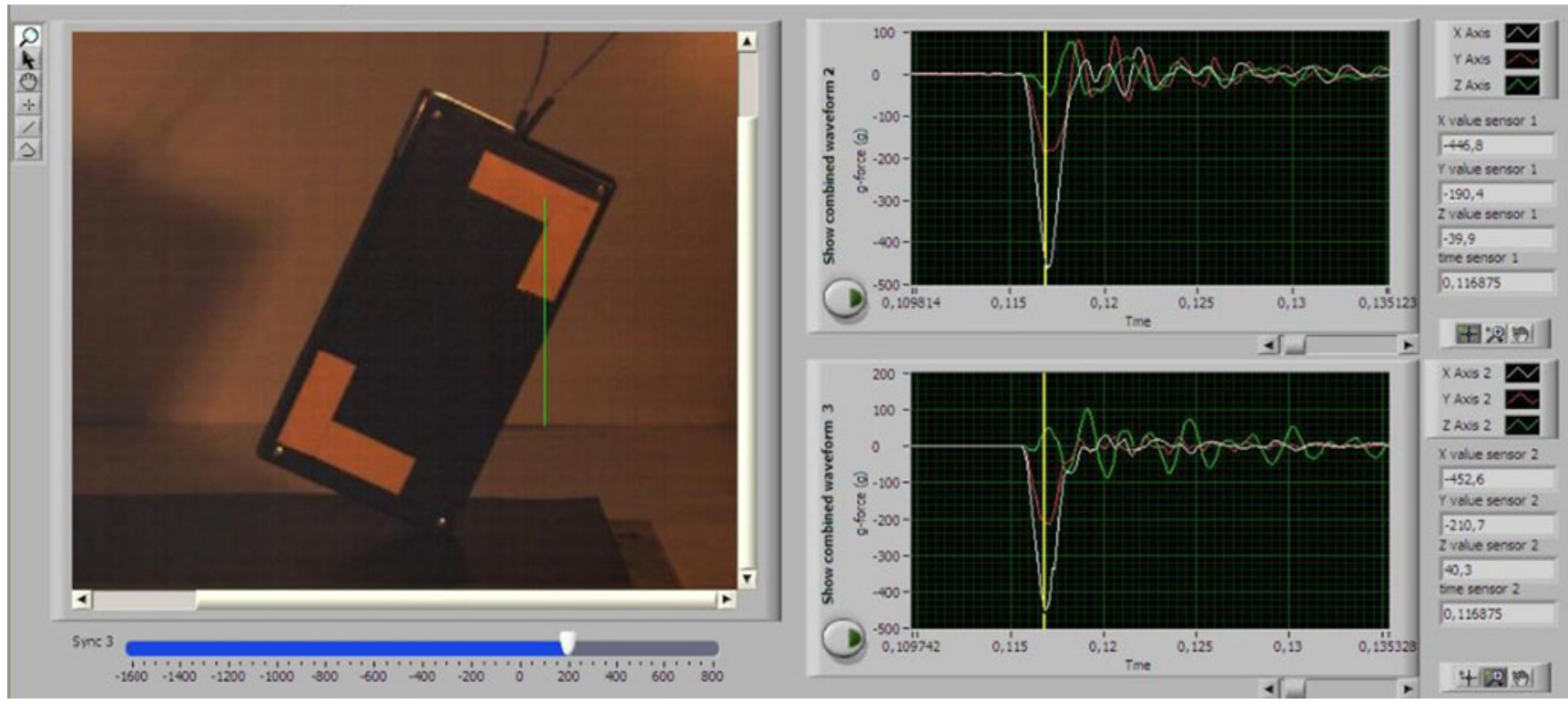

Fig. 7 Typical acceleration histories (right: S2 data top, S1 data bottom) for geometry-1 at $25^{\circ}$, plus HS video image (left). Vertical yellow lines in histories denote midway during the contact time

For geometry-2A, the results are given in Table 2, with typical acceleration histories in Fig. 8. Comparing the data streams, the time interval between the peaks for S1 (centred on rubber-mounted plate) and S2 (near top inside case) was found to be $2.0 \mathrm{~ms}$. From the histories, an oscillation frequency for the shock-mounted plate was found of $129 \mathrm{~Hz}$ (averaged over first three oscillations). Similarly, Table 3 and Fig. 9 present the results for geometry-2B. Here, the time interval between the peaks for $\mathrm{S} 1$ and $\mathrm{S} 2$ was found to be $1.2 \mathrm{~ms}$ and an

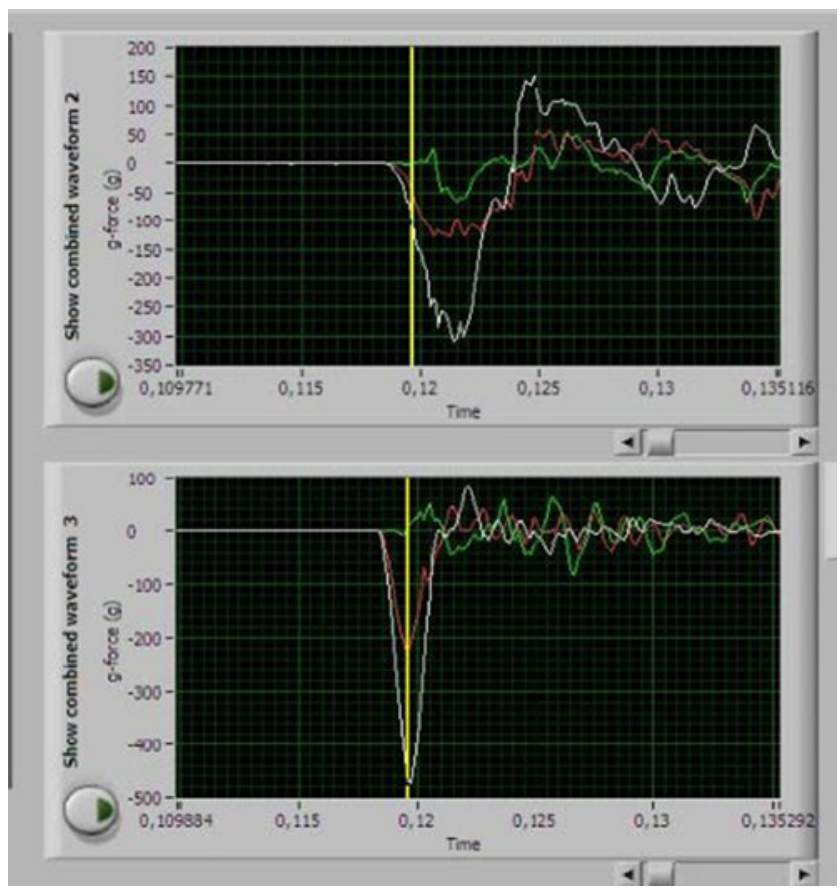

Fig. 8 Typical histories, geometry-2B (S2 data top, S1 data bottom) oscillation frequency for the plate was found of $177 \mathrm{~Hz}$ (again averaged over first three oscillations).

As for characterization, the force-displacement curve for the supported plate in geometry-2A was found to be linear for displacements between $0.1-1.0 \mathrm{~mm}$, with the plate's lateral stiffness in the XY-plane being $k_{p}=7.55 \mathrm{KN} / \mathrm{m}$. Furthermore, this stiffness was found to be independent of the orientation. The Young's modulus of the ABS was found to

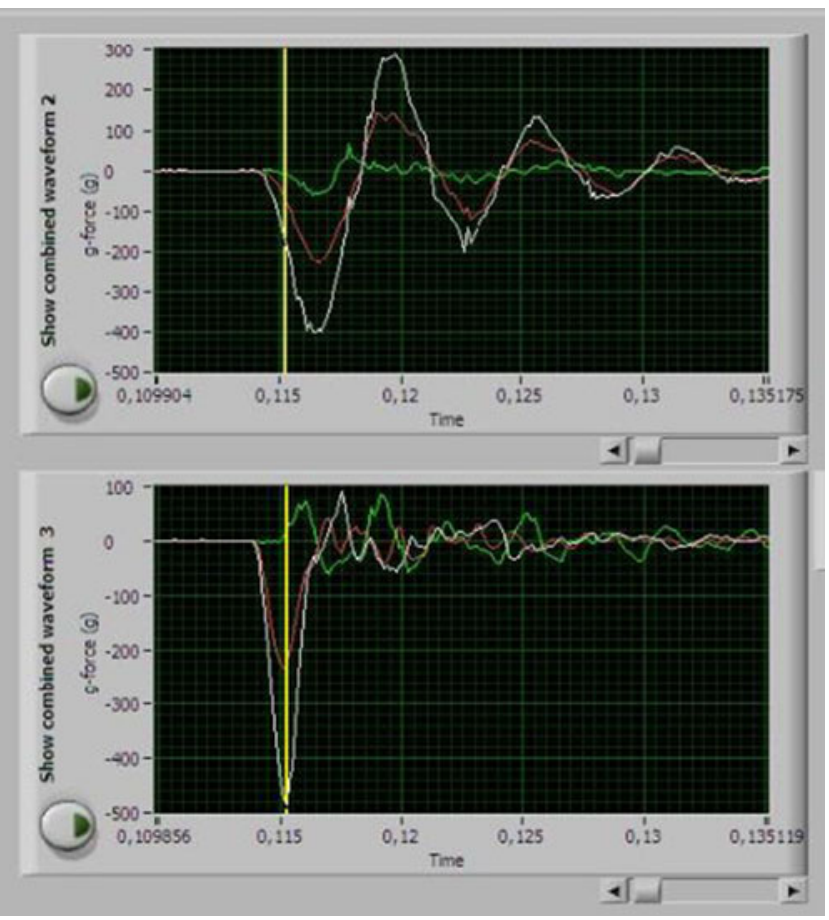

Fig. 9 Typical histories, geometry-2B (S2 data top, S1 data bottom) 
Table 2 Sensor data, geometry-2A

\begin{tabular}{|c|c|c|c|c|c|c|c|c|c|c|}
\hline & & \multicolumn{4}{|c|}{ Sensor-1 (centred on plate) } & \multicolumn{4}{|c|}{ Sensor-2 (near top inside case) } & \multirow[t]{2}{*}{ unit } \\
\hline & & $\mathrm{X}$ & $\mathrm{Y}$ & $\sqrt{ }\left(X^{2}+Y^{2}\right)$ & $\mathrm{Z}$ & $\mathrm{X}$ & $\mathrm{Y}$ & $\sqrt{ }\left(X^{2}+Y^{2}\right)$ & $\mathrm{Z}$ & \\
\hline \multirow[t]{2}{*}{ angle 15} & mean & $-347,2$ & $-129,2$ & 370,5 & $-28,2$ & $-536,4$ & $-256,4$ & 594,5 & $-9,7$ & $\mathrm{~g}$ \\
\hline & SD & 9,7 & 19,4 & & 3,5 & 3,8 & 14,5 & & 14,5 & $\mathrm{~g}$ \\
\hline \multirow[t]{2}{*}{ angle 20} & mean & $-352,6$ & $-118,7$ & 372,0 & $-23,0$ & $-507,6$ & -241 & 561,9 & 1,7 & $\mathrm{~g}$ \\
\hline & SD & 30,2 & 4,7 & & 9,0 & 6,1 & 4,5 & & 2,2 & $\mathrm{~g}$ \\
\hline \multirow[t]{2}{*}{ angle 25} & mean & $-310,1$ & -133 & 337,4 & $-30,0$ & $-485,1$ & $-218,4$ & 532,0 & 6,7 & $\mathrm{~g}$ \\
\hline & SD & 24,2 & 25,8 & & 3,1 & 8,1 & 8,6 & & 1,1 & $\mathrm{~g}$ \\
\hline \multirow[t]{2}{*}{ angle 30} & mean & $-339,5$ & $-176,2$ & 382,5 & $-35,8$ & $-471,9$ & $-200,4$ & 512,7 & 7,4 & $\mathrm{~g}$ \\
\hline & SD & 6,9 & 3,3 & & 6,6 & 3,8 & 6,2 & & 1,5 & $\mathrm{~g}$ \\
\hline \multirow[t]{2}{*}{ angle 35} & mean & -325 & $-169,4$ & 366,5 & $-39,2$ & $-455,2$ & $-195,7$ & 495,5 & 5,7 & g \\
\hline & SD & 8,2 & 3,6 & & 3,8 & 17,8 & 12,1 & & 2,3 & $\mathrm{~g}$ \\
\hline
\end{tabular}

be $1.14 \mathrm{GPa}$ (at $5-25 \%$ of the tensile strength) to $1.10 \mathrm{GPa}$ (at $10-40 \%$ ).

Overlapping images taken midway at the contact time revealed that angle control was very good. Figure 6 shows a typical image of overlapping five separate drop tests (taken for geometry- 1 , at $35^{\circ}$ angle). In the figure, the five different images of the product captured from five different tests actually coincide almost perfectly: only the wires were in different locations during each individual drop. Finally, a rebound ratio of $e_{c}=50 \%$ was recorded.

\section{Discussion}

With a contact time of $3 \mathrm{~ms}$, a sample rate of $7 \mathrm{kHz}$ (X-axis) means that just 21 actual acceleration measurements were recorded during that time, and a first 'reality check' using elementary physics is in order. With the set drop height and the observed rebound ratio $e_{c}$, a velocity change of $\Delta V=6.28 \mathrm{~m} / \mathrm{s}$ coincides with the test results. Assuming that the accelerations build up sinusoidally from zero to the recorded peak value during the first half of the contact time, as seems reasonable from the acceleration histories, a peak deceleration of $427 \mathrm{~g}$ can be predicted. This matches the order of magnitude of the results quite well. In addition, with few exceptions, the standard deviations are small compared to the actual peak values $(<5 \%)$. The overlapping drop images (Fig. 6) further increase confidence in the reliability and reproducibility of the results. Note that at 500-600g, the sensors get outside their designed measurement range, but are still quite reliable.

At the COG of geometry-1, peak acceleration along the $\mathrm{X}$-axis drops with increasing contact angle, while peak acceleration along the $\mathrm{Y}$-axis increases. At the location of S2 (65 mm up), the trend is different: decreasing with angle both for the X-and the Y-component. This last trend is seen for all three geometries and suggests that at larger angles, a significant part of the impact energy is directed away from the area near S2. All in all, this behaviour is realistic and explicable, at least in the semi-quantitative sense. This concludes the reality check.

Table 3 Sensor data, geometry-2B

\begin{tabular}{|c|c|c|c|c|c|c|c|c|c|c|}
\hline & & \multicolumn{4}{|c|}{ Sensor-1 (centred on plate)) } & \multicolumn{4}{|c|}{ Sensor-2 (near top inside case) } & \multirow[t]{2}{*}{ unit } \\
\hline & & $\mathrm{X}$ & $\mathrm{Y}$ & $\sqrt{ }\left(X^{2}+Y^{2}\right)$ & $\mathrm{Z}$ & $\mathrm{X}$ & $\mathrm{Y}$ & $\sqrt{ }\left(X^{2}+Y^{2}\right)$ & $\mathrm{Z}$ & \\
\hline \multirow[t]{2}{*}{ angle 15} & mean & $-420,7$ & $-186,9$ & 460,3 & $-28,4$ & $-627,4$ & $-302,4$ & 696,5 & 0,1 & $\mathrm{~g}$ \\
\hline & SD & 10,2 & 16,6 & & 3,5 & 22,1 & 14,1 & & 2,7 & $\mathrm{~g}$ \\
\hline \multirow[t]{2}{*}{ angle 20} & mean & $-410,8$ & $-224,5$ & 468,1 & $-33,3$ & $-517,9$ & $-238,2$ & 570,1 & 5,4 & $\mathrm{~g}$ \\
\hline & SD & 7,2 & 2,1 & & 1,7 & 13,1 & 8,3 & & 0,7 & $\mathrm{~g}$ \\
\hline \multirow[t]{2}{*}{ angle 25} & mean & -401 & $-225,9$ & 460,3 & $-31,5$ & $-475,2$ & $-220,2$ & 523,7 & 9,4 & $\mathrm{~g}$ \\
\hline & SD & 18,4 & 4,6 & & 3,4 & 10,0 & 5,0 & & 1,4 & g \\
\hline \multirow[t]{2}{*}{ angle 30} & mean & $-397,6$ & $-245,9$ & 467,5 & $-30,6$ & $-445,8$ & $-198,1$ & 487,8 & 8,6 & $\mathrm{~g}$ \\
\hline & SD & 5,1 & 3,8 & & 4,3 & 9,5 & 5,0 & & 0,5 & g \\
\hline \multirow[t]{2}{*}{ angle 35} & mean & $-382,1$ & $-245,0$ & 453,9 & $-31,6$ & $-417,5$ & $-184,3$ & 456,4 & 8,2 & $\mathrm{~g}$ \\
\hline & SD & 5,3 & 3,5 & & 3,5 & 4,8 & 3,8 & & 1,3 & g \\
\hline
\end{tabular}


Table 4 Comparison of S1 data across geometries

\begin{tabular}{|c|c|c|c|c|c|c|}
\hline & \multicolumn{3}{|c|}{ Geometry-2A vs geometry-1 } & \multicolumn{3}{|c|}{ Geometry-2B vs geometry-1 } \\
\hline & \multicolumn{3}{|c|}{$\mathrm{S} 1_{\text {geo-2A }} / \mathrm{S} 1_{\text {geo-1 }}$} & \multicolumn{3}{|c|}{$\mathrm{S} 1_{\text {geo-2B }} / \mathrm{S} 1_{\text {geo-1 }}$} \\
\hline & $\mathrm{X}$ & $\mathrm{Y}$ & $\sqrt{ }\left(X^{2}+Y^{2}\right)$ & $\mathrm{X}$ & Y & $\sqrt{ }\left(X^{2}+Y^{2}\right)$ \\
\hline angle 15 & 0,60 & 0,68 & 0,61 & 0,73 & 0,98 & 0,76 \\
\hline angle 20 & 0,70 & 0,56 & 0,68 & 0,82 & 1,05 & 0,86 \\
\hline angle 25 & 0,67 & 0,71 & 0,67 & 0,86 & 1,20 & 0,92 \\
\hline angle 30 & 0,76 & 0,75 & 0,76 & 0,89 & 1,05 & 0,93 \\
\hline angle 35 & 0,77 & 0,67 & 0,74 & 0,90 & 0,96 & 0,92 \\
\hline
\end{tabular}

If the resultant in-plane vectors $\sqrt{ }\left(X^{2}+\mathrm{Y}^{2}\right)$ as measured at the location of S2 are compared across geometries, they are found to be very similar $\left(\mathrm{S} 2_{\text {geo- } 1} / \mathrm{S} 2_{\text {geo-2A }}=1.00-0.95\right.$ and $\left.\mathrm{S} 2_{\text {geo- } 1} / \mathrm{S} 2_{\text {geo-2B }}=0.95-0.99\right)$. This means first, that the inplane peak accelerations in the box at the location of S2 are not significantly affected by the presence of the rubbermounted plate, and second, that the measurements at the location of S1 across geometries can also be compared. This last comparison is made in Table 4 . In this particular situation, the 3-mm cubes are more effective as shock mounts than the 5-mm cubes. This (at first sight counterintuitive) finding is supported by the different oscillation frequencies (129 vs. $177 \mathrm{~Hz}$ ) and the different time intervals between peaks at S1 and S2 (2.0 vs. $1.2 \mathrm{~ms})$ that were measured.

As for the out-of-plane behaviour i.e. accelerations in the Z-direction, Tables 1, 2 and 3 show that these are small compared to the in-plane behaviour; with one exception (= geometry $1,15^{\circ}$ angle, sensor 2 ), the standard deviations on these values were small as well. For geometry-1, out-ofplane accelerations were typically $<5 \%$ of the X-acceleration. Notice that the values were negative at the $\mathrm{COG}$ and positive near the top, which probably means that for this geometry, there was slight rotation around the Y-axis, most likely during contact (although this was not unmistakably observed in the video). Geometry-2 showed a different trend, with somewhat larger Z-accelerations on the plate and clearly smaller values near the top. Still, compared to the in-plane accelerations, these values remain small (typically $<10 \%$ ), which supports our choice to model only in-plane motion.
The question is now if these measurements can be reproduced using the relatively simple theoretical model presented earlier. Given that the rubber-mounted plate in geometry-2A has a constant shear stiffness $k_{p}=7.55 \mathrm{KN} / \mathrm{m}$ in the XY-plane, independent of the angle, the assumption seems valid that the main deformation mechanism is simple shear, which also infers that the natural frequency in any direction is constant and is a function of the cube's side length. Then, the cube's stiffness scales linearly with this length. Assuming that the mode of deformation of the cubes is governed by shear and that the shear strain is constant along the cube height, the lateral stiffness can be computed as:

$k_{p}=\omega_{p}^{2} m_{p}=\frac{4\left(\tau \times t_{r}^{2}\right)}{\left(\frac{\tau}{G}\right) \times t_{r}}=4 G t_{r}$

Here $\tau$ is the shear stress, $t_{r}$ is the side length of the rubber cube, and $G$ is the shear modulus of the rubber used in the drop tests. Using equation (19), this modulus is determined to be $G=0.63 \mathrm{MPa}$. Using Poisson's ratio of $\mu_{r}=0.499$, the Young's modulus is then found to be $E_{r}=1.88 \mathrm{MPa}$. Using these data, the natural frequency of the mounted plate was thus computed as $\omega_{p}=\sqrt{4 G t_{r} / m_{p}}$. This method predicts the natural frequency for geometries $2 \mathrm{~A}$ and $2 \mathrm{~B}$ as 121 and $154 \mathrm{~Hz}$, respectively. Given the simplicity of the method, this prediction compares well with the averaged values obtained directly from experiments (129 and $177 \mathrm{~Hz}$, respectively).
Fig. 10 (a)-(b): Measured (point) and predicted (point plus line) peak accelerations,

$\mathrm{X}$-direction

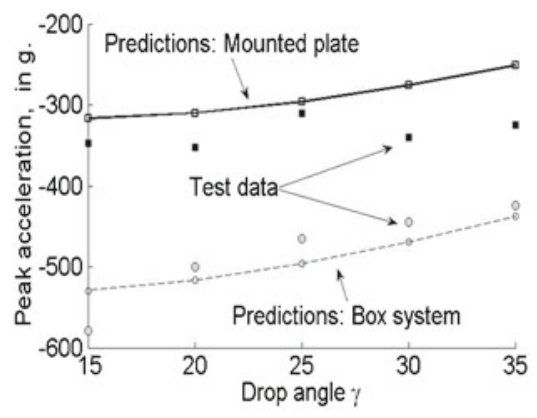

(a) Geometry-2A (3mm cubes)

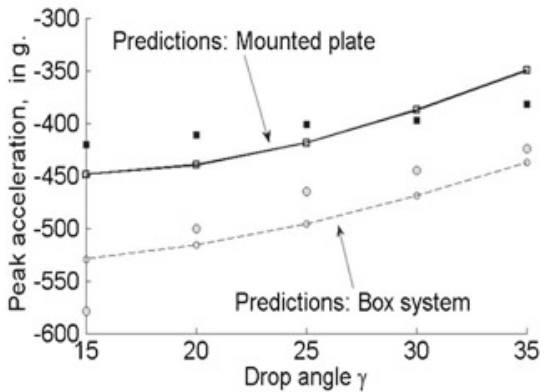

(b) Geometry-2B (5mm cubes) 
Fig. 11 (a)-(b): Measured (point) and predicted (point plus line) peak accelerations, Y-direction

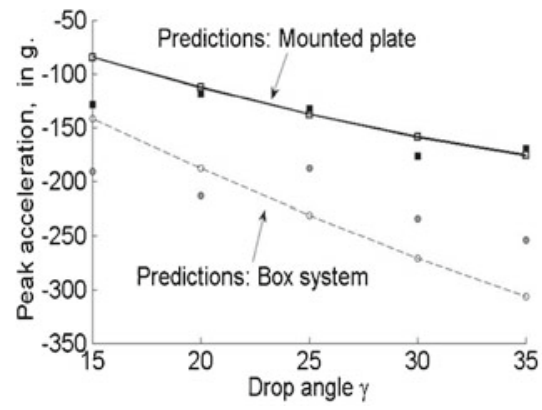

(a) Geometry-2A (3mm cubes)

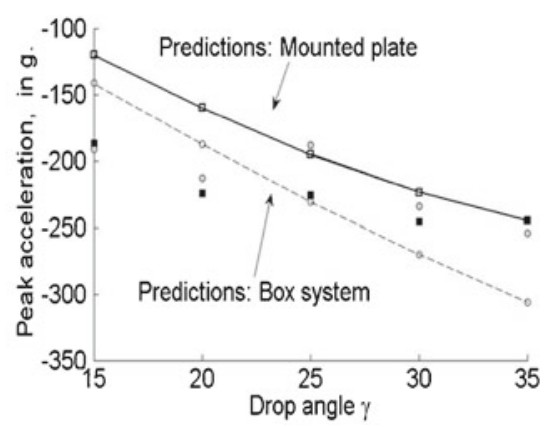

(b) Geometry-2B (5mm cubes)
From the recorded accelerations and using logarithmic decrement of the dynamic response, the damping ratio of rubber and the box system were taken as $\xi_{p}=0.1$ and $\xi_{B}=$ 0.4 . The radius of impact $R$ is assumed to be $1.5 \mathrm{~cm}$, which is close to the largest dimension of the box that made contact with the rubber mat. The Young's modulus of the ABS plastic was measured to be 1.10-1.14 GPa with $\mu_{A B S}=0.451$.

The input data mentioned above are fed into the equations derived earlier in this study, generating predictions of the peak X- and Y-accelerations for geometries-2A and -2B. These are plotted in Figs. 10a-b and 11a-b. Likewise, the acceleration histories were predicted as a function of time and are shown in Figs. 12a-b. To facilitate comparison, the measured values are also included in the Figs. 10, 11. Comparison shows that with reasonable accuracy, the proposed model predicts the trends in recorded peak accelerations and histories for all cases.

Figure 11 shows in general good agreement between theoretical and experimental values of accelerations. The deviations between model predictions and measured values in Fig. $11 \mathrm{~b}$ could be related to the assumption that the rubber material behaves perfectly elastic. This assumption is made in order to arrive at a simplified solution of the impact problem because the analysis is proposed for the conceptual phase of the design process. In reality, however, rubber behaves in a hyperelastic fashion and the "stiffness" properties of the rubber are dependent on the rate of loading. Based on this reasoning, it can be that the assumed stiffness of rubber is closer to the actual rubber stiffness under typical high strain rates that occurred in the X-direction (thus Fig. 11a gives better agreement than Fig. 11b). This is supported by the fact that the error between measured and predicted accelerations is smaller as the value of the Yacceleration is higher in Fig. 11b. Another reason that can cause deviations between experimental and theoretical values is due to simplification of the natural frequency computation of the mounted plate. It was assumed that the in-plane stiffness is mainly due to shear deformation of the rubber cube, but as the cubes become bigger, other mode of deformation, such as flexural, start to become more significant.

In total, the differences between predictions and measured values can be attributed to the simplifications made in the model and to the unknown parameters that were assumed in the analysis, such as the damping ratios, stiffness (material and structure), and radius of contact. Note also that this study neglects the influence of strain rate; this is to simplify the analysis and make it applicable to conceptual design. Finally, small variations can be attributed to slight inaccuracies that remained in the experiments, such as the exact sizes of the rubber cubes.

Overall, the proposed method, on its simplicity, seems to be a useful tool to predict trends and peak values of accelerations in products of similar structure and configuration during drop impact. The comparisons highlight that the simplified procedure for analysis proposed here is a decent tool for preliminary design of base-isolation systems aimed
Fig. 12 (a)-(b): Acceleration history at location S1 (at COG, on box system and on mounted plate), geometry-2A (3 $\mathrm{mm}$ cubes), drop angle $25^{\circ}$

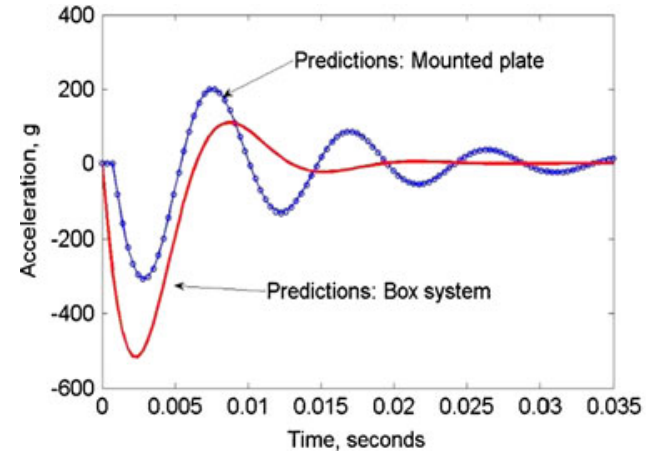

a) X-direction

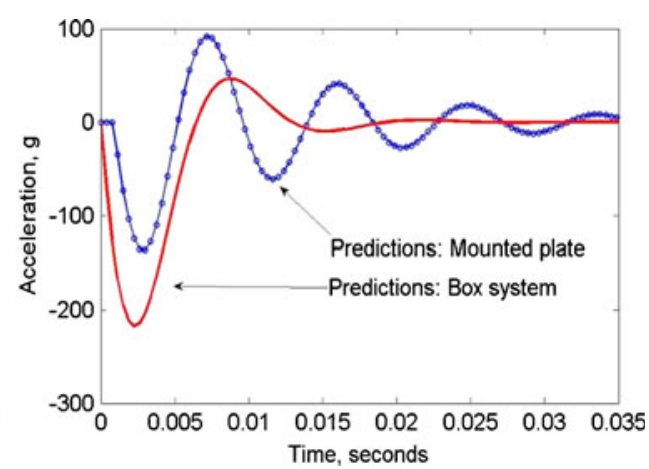

b) Y-direction 
at reducing the peak accelerations during drop impact of portable products. Of course, more rigorous analysis and FEM modelling using computers would be required during later phases of the design process, but until that time, analytical tools clearly have their strengths and uses, particularly if backed up with small-scale experiments to measure $k_{p}$. Apart from this, the drop test facility used in this study shows its usefulness as an experimental tool: the guide-andrelease set-up employed allows reproducible drops across a range of contact angles, in accordance with the pioneering work of Goyal \& Buratynski [9] and the improvements made by Lim et al. [7]. The use of twin accelerometers as presented here appears to be a novel and promising extension of their work, particularly since it allows systematic study of product variables with respect to impact resistance. Note that high speed video remains indispensable to making reliable measurements.

\section{Conclusions}

A special drop tower has been built that allows accurate control over the contact angle for product drop tests, even with wire-based sensors placed in or on the test product. The work reaffirms the suitability of guide-and-release facilities in impact testing, underlines the potential of combining high speed video with the use of built-in sensing, and opens the door to systematic impact testing with multiple sensors placed in various locations of the product. The set-up has been successfully applied to study the effect of internal rubber shock mounting in simplified product geometries, specifically to the reductions in shock intensity that such mounts can give. In the analytical side of this work it was found that these values can be adequately predicted for a range of contact angles on the basis of a simple, SDOF analytical model, in which only the rubber mounts are assumed to deform. Of course, for more complex geometries, computer modelling will be indispensable, but if it is possible to treat the product casing as infinitely-rigid, then such modelling can be relatively lightweight. This facilitates knowledge-driven design for impact resistance already during the first, conceptual stages of product design.
Acknowledgements Ridzert Ingenegeren, Luc Bolier, Roosmarijn Schopman and Antonio Trolio (at the time of writing, all were MSc students at IDE faculty, TU Delft) and Herman Broekhuizen (staff member, IDE, TU Delft) are acknowledged for making improvements to the drop test facility, data acquisition and initial interpretation, and suggestions for experiments.

Open Access This article is distributed under the terms of the Creative Commons Attribution Noncommercial License which permits any noncommercial use, distribution, and reproduction in any medium, provided the original author(s) and source are credited.

\section{References}

1. Amy RA, Aglietti GS, Richardson G (2009) Reliability analysis of electronic equipment subjected to shock and vibration - a review. Shock Vib 16(1):45-59

2. Goyal S, Upasani S, Patel DM (1999) Improving impact tolerance of portable electronic products: case study of cellular phones. Exp Mech 39(1):43-52

3. Wonga EH, Seah SKW, van Driel WD, Caers JFJM, Owens N, Lai Y-S (2008) Advances in the drop-impact reliability of solder joints for mobile applications. Microelectron Reliab 29(2):139-149

4. Zaal JJM, van Driel WD, Kessels FJHG, Zhang GQ (2009) Correlating drop impact simulations with drop impact testing using high-speed camera measurements. J Electronics Packaging 131(1)

5. Kwon S, Lee B, Park S (2007) Investigation of the failure of a liquid crystal display panel under mechanical shock. Proc ImechE, Part C J Mech Eng Sci 221(11):1475-1482

6. Zhou CY, Yu TX, Lee RSW (2008) Drop/impact tests and analysis of typical portable electronic devices. Int J Mech Sci 50(5):905917

7. Lim CT, Ang CW, Tan LB, Seah SKW, Wong EH (2003) Drop impact survey of portable electronic products, Proceedings of the IEEE 2003 Electronic Components and Technology Conference, New Orleans, USA, 27-30 May 2003

8. Singh R, Mattoo A, Saigal A (2006) Optimizing the design and impact behavior of a polymeric enclosure. Mater Des 27(10):955967

9. Goyal S, Buratynski EK (2000) Methods for realistic drop-testing. The International Journal of Microcircuits and Electronic Packaging 23(1)

10. Love AHE (1944) A treatise on the mathematical theory of elasticity. Dover, New York, pp 193-203

11. Hunter SC (1957) Energy absorbed by elastic waves during impact. J Mech Phys Solids 5(3):162-171

12. Beards CF (1996) Structural vibration: analysis and damping. Arnold, Hodder Headline Group, London 